\title{
Star Forming Rates and HI Gas Content in Galaxy Groups
}

\author{
Sarah Martens \\ Department of Astronomy, University of Wisconsin- Madison
}

We compare the star formation rates with the respective HI properties of 4854 galaxies in 743 different groups. Using data from the SDSS and the GALEX all sky survey (AIS) we measure the star formation rates and NUV-r colors as a function of the total group mass, position within the group, and HI content as derived from the Arecibo Legacy Fast ALFA (ALFALFA) survey. The NUV-r colors are consistent with star forming field galaxies. We show how the quenching of the star formation is correlated with galaxy HI content and how both are affected by a group environment. ${ }^{\mathrm{i}}$

\section{Introduction}

Galaxies residing in group environments have been shown to experience different evolutionary patterns than galaxies that are not in such tidally strong environments (Hess\&Wilcots, 2013).

Features such as NUV r color and HI mass can be analyzed to create an image of stellar population in galaxies with respect to their HI mass, two values known to be inversely proportional (as gas is the fuel to produce stars), at least in isolated environments. Studying how these values change in each galaxy with respect to their distance from the center of the group, we show how group environments affect galaxy star formation rates and gas content, leading to a possible loss of HI gas causing halt in star formation, or quenching. In Hess\&Wilcots 2013 it was found that $\mathrm{HI}$ detections in galaxies decrease as the number of members in a group increases. Others found that galaxies, when organized in NUV-r color vs. $r$ magnitude plots, tend to gather in a blue cloud of galaxies with a younger-forming stellar populations and a red sequence containing galaxies that have been recently quenched and therefore have an older stellar population than the former (Schawinkski et al., 2014). There has been speculation of there being a third grouping, the green valley, of galaxies independent of both the red sequence and blue cloud, however this has been found to be only a transitional period between youthful energetic galaxies and older quenched galaxies with larger stellar populations (Schawinkski et al., 2014).

We utilize three sources of astronomical data to perform our study. Sloan Digital Sky Survey (SDSS) provides the original sample used in Hess\&Wilcots of 4854 different galaxies and the respective data in the optical. We then used the GALEX catalog to obtain the UV data of the galaxies from our original sample that could be detected. With this new sample we incorporate the galaxies HI data from the Arecibo Fast Legacy ALFA Survey (ALFALFA Survey). In this

\footnotetext{
i Thank you to the Undergraduate ALFALFA TEAM with NSF grant AST-1211005, Wisconsin Space Grant Consortium, and The National Space Grant College and Fellowship Program NASA Training Grant \#NNX14AP22H
} 
paper we present an analysis of HI mass detections from Hess\&Wilcots and star formation rates as a function of relative distance of a galaxy from the group center. This paper is organized as follows. In Section 2 we discuss our three data sources: the optical data from the SDSS Data Release 7, the NUV and FUV magnitudes and luminosities derived from GALEX data, and the HI from the ALFALFA Survey. We also discuss the limiting of our sample by galaxies that are non UV-detected and the sky coverage of this sample. In Section 3 we analyze the significance of our results in the scope of the evolution of galaxies in group environments and in Section 4 we draw conclusions from these implications.

\section{Data}

Our original data is based upon the group sample that is described thoroughly in Hess\&Wilcots(2013). Sloan Digital Sky Survey provided data ranging from 114.199844 to 247.473602 in right ascension and from 3.808206 to 29.015427 in declination. The size of this original sample in the optical was decreased when we began to incorporate GALEX data. Many of the galaxies from our original weren't detected in the UV. With this new sample we then compared their HI masses using the ALFALFA survey data from Hess\&Wilcots 2013.

2a. SDSS: We obtained the original sample of galaxies involved in our investigation from Sloan Digital Sky Survey DR 7 which defined group galaxies by the friends of friends algorithm as described in Hess\&Wilcots 2013, which can be referenced for a more detailed description of the original sample. We began our investigation with 4854 galaxies in 743 different groups ranging from 3 to 525 members in a group. This data contained information on right ascension and declination along with redshift. We used the redshift SDSS values to calculate the distances of these original galaxies using Hubble's Law, and we were later able to find the relative distances of each of the galaxies to the center of their respective group in Mpc. Using the HI data from ALFALFA, this allowed us to study the relationship of HI mass and star formation as a function of distance from group center, using distance from the center as a proxy for the time in which a galaxy would remain a member of a group.

2b. GALEX: The orbiting space telescope, GALEX, provided the ultraviolet data to contribute to our study. Our sample was limited slightly because of a portion of the galaxies detected by SDSS went undetected by GALEX. In order to determine which galaxies from SDSS were UV detected we had to search for matches around each of the original SDSS measurements. We chose 0.0001 as the range the differences between the right ascension and declination of both sources could be in order to be considered a match. The matched data was divided amongst the multiple surveys in the GALEX catalog the Medium Imaging (MIS), All-Sky Imaging (AIS), and Nearby Galaxy (NGS) of which we chose the most complete, the AIS, to be our modified sample of UV detected galaxies. The All Sky Imaging Survey (AIS) was done to by necessity to maintain the largest and most consistent and cohesive sample possible. The galaxies from our original SDSS data that were detected by GALEX experienced more star formation in there recent history, as the highly energetic light produced by young stellar populations are what allow the galaxies to be UV detected. From data provided by the AIS we were able to calculate the absolute magnitudes and luminosities of the galaxies, the equations used can be viewed in the Section 3. These new values enabled us to analyze the difference of the NUV-r color of galaxies with respect to their r-magnitudes and their distance from the center of their respective group. 
This relationship allows us to observe how star formation rates, respond to the intensity of a group environment based on proximity.

2c. ALFALFA: The ALFALFA survey is an HI profiling survey from Arecibo Observatory which has the ability to detect low mass and volume HI content to $10^{6}$ solar masses and has a positional accuracy of approximately 20 arc seconds) in the local universe (Haynes, Martha). HI data from the 2011 ALFALFA sample, which covered 40 percent of the complete survey area, contributes essentially to our analysis of star formation and quenching of galaxies in group environments as it represents the potential a galaxy has to producing more stars. The data we received from the ALFALFA survey allows for further analysis of galaxies evolution contributing to the existing optical (SDSS) and UV (GALEX) data to develop a deeper understanding of the effects of group and clustered environments on the stellar formation and gas content. For a more detailed description of the survey see Haynes et al. 2011.

2d. Sky Coverage and HI Detections: We used the Hess\&Wilcots 2013 sample of 4500 galaxies in 754 different groups from SDSS ranging in the right ascension and declination measurements stated earlier. Of this original sample groups of less than 3 members had been deemed ineffective to study because forces due to the group dynamic may not be as distinguishable from a non-group environment or as consistent as with the larger more stable groups. Sky coverage was in part limited by the All-Sky Imaging Survey (AIS) from GALEX which we chose as the most complete survey provided by GALEX (as discussed in Section 2b). In Hess\&Wilcots 2013 the original right ascension and declination measurements from SDSS where matched with those given in the ALFALFA Survey containing the HI data in order to determine which galaxies had detected gas mass. For more information on the sky coverage of the sample reference Hess\&Wilcots 2013.

\section{Results}

Beginning with a sample of galaxies, the respective groups they resided in, along with their positional and optical data from SDSS we used CasJobs query to retrieve the respective far and near UV data for all of the galaxies. After organizing the UV and optical data we then converted our apparent NUV and FUV magnitudes in to absolute magnitudes. We then used them to calculate the near and far UV luminosities using a Python code. The following equations were used where FUV is the apparent far UV magnitude, $\mathrm{c}$ is the speed of light, $\mathrm{z}$ is the redshift, and MFUV is the absolute far UV magnitude.

$$
\begin{gathered}
M F U V=(F U V-5)\left(\log \left(\frac{C * Z}{7}\right)\right) \\
\text { Luminosity } F U V=\left(3.846 * 10^{26}\right)\left(e^{\frac{M F U V-4.47}{-2.5}}\right)
\end{gathered}
$$


The same equations above were used to find the absolute magnitude and luminosity of the near UV data. With this more complete and informative data, we made plots using TOPCAT (Tool for Operations on Catalogs and Tables) software of the absolute NUV magnitude as a function of the r-band magnitude from SDSS. These plots allow us to discuss the number of stars in a galaxy and their ages, looking at blue cloud vs. red sequence galaxies. Figure 1 is our complete sample plotted in this manner. Very few galaxies that have a very low $r$ magnitude (high luminosities) are detected. The high luminosity galaxies that are detected have a lower UV output. this conclusion can be drawn intuitively because galaxies that aren't detected in the optical ranges, which have lower energy, will surely then not be detected in a higher energy wavelength.

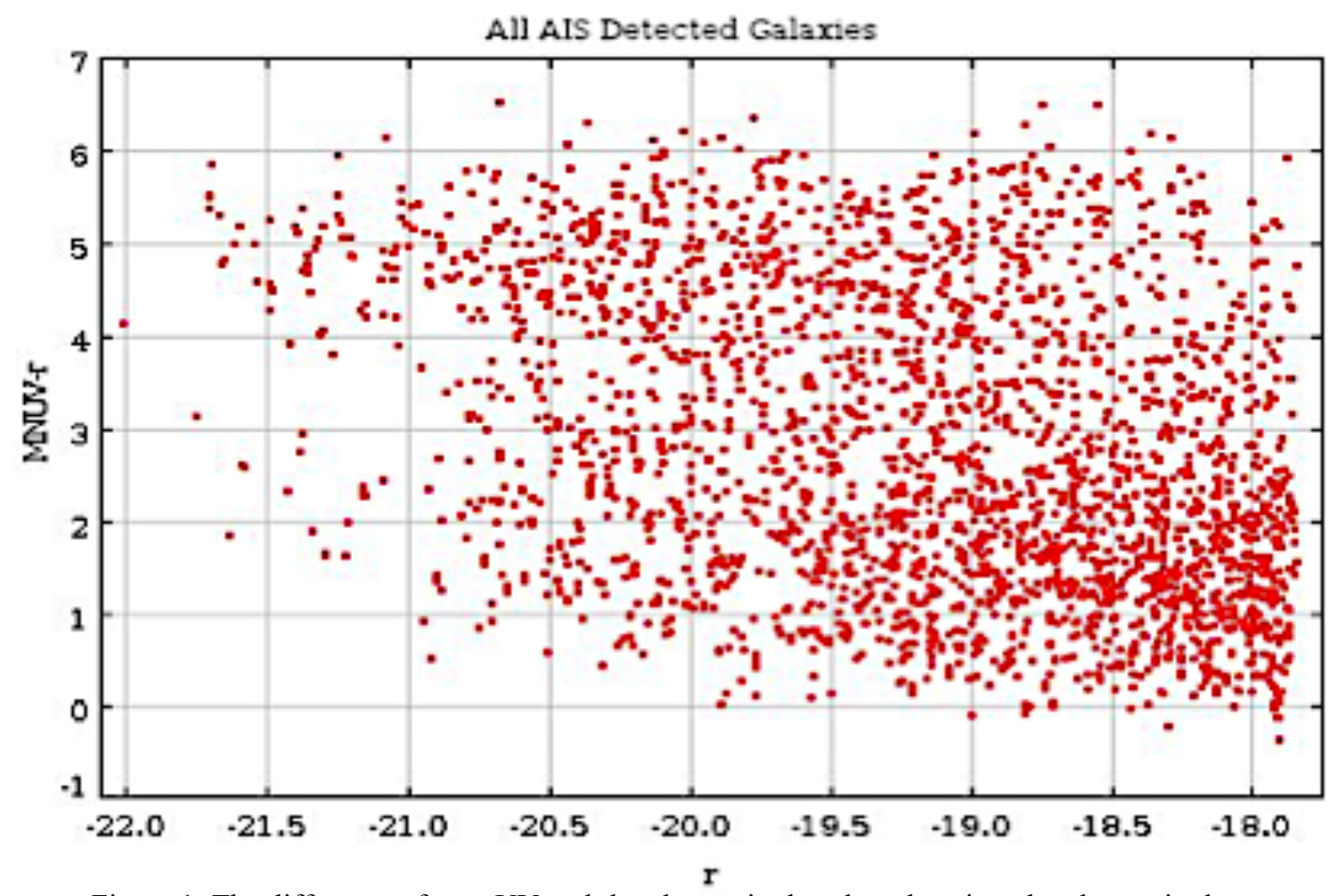

Figure 1: The difference of near UV and rband magnitudes plotted against rband magnitudes for all UV detected galaxies in the sample.

However, moving toward the higher $r$ magnitudes (lower luminosities) there is a higher UV output. Also there is somewhat of a gradient of galaxy concentration with the lowest concentration in the area of smaller $r$ magnitude and higher NUV (corresponding to the red sequence galaxies) and the more densely populated region being of high magnitude and low NUV (corresponding the blue cloud). This lends itself to the idea that galaxies within group environments undergo rapid star formation due possibly to strong tidal forces collapsing gas reserves. The many blue cloud galaxies, and the sparseness of red sequence supports this idea of ongoing quenching within group environments as blue cloud galaxies become quenched.

In order to analyze the spatial distribution of UV detected and HI mass galaxies with respect to the center of their groups we had to convert our original RA and DEC measurements to Mpc using the following equation: 


$$
R a M p c=\frac{(R a-\operatorname{GroupRa})\left(\frac{c * Z}{70}\right)}{(206265 * 3600)}
$$

The same equation was used with respect to finding the declination in $\mathrm{Mpc}$ from the center of the group. After plotting the SDSS spatial distributions of the galaxy groups we then plotted the spatial distributions of the UV and HI detected galaxies on top in order to discuss a possible relationship between distance from the group center and UV and HI magnitude Figure 2. The HI data provided the known relationship between the stellar mass and gas content (in general in an isolated system will show an inverse relationship as stellar formation requires gas content) and allowed us to analyze further the possibility of quenching in galaxy groups. The more galaxies in each group that are UV detected, especially near the center will increase evidence for galaxy quenching at large, as seen in Figures 2. Meaning that galaxies residing in groups initially will experience a high star formation rates and gas depletion, leaving them energetic with a large stellar population but with no future potential of high levels of star formation, leaving them to become red sequence galaxies. In reference to red diamonds in the figures, we see an extreme depletion of galaxies having $\mathrm{HI}$ as group membership increases (similarly to Hess\&Wilcots), especially nearer to the center of the group. The results of these plots coincide well with the interpretation of the UV distributions because the heavy UV detections nearly the center are rich in new stars but have no more HI reserves. This also heavily supports the theory of the quenching of gas in galaxies in groups due to tidal and gravitational forces increasing with higher membership.

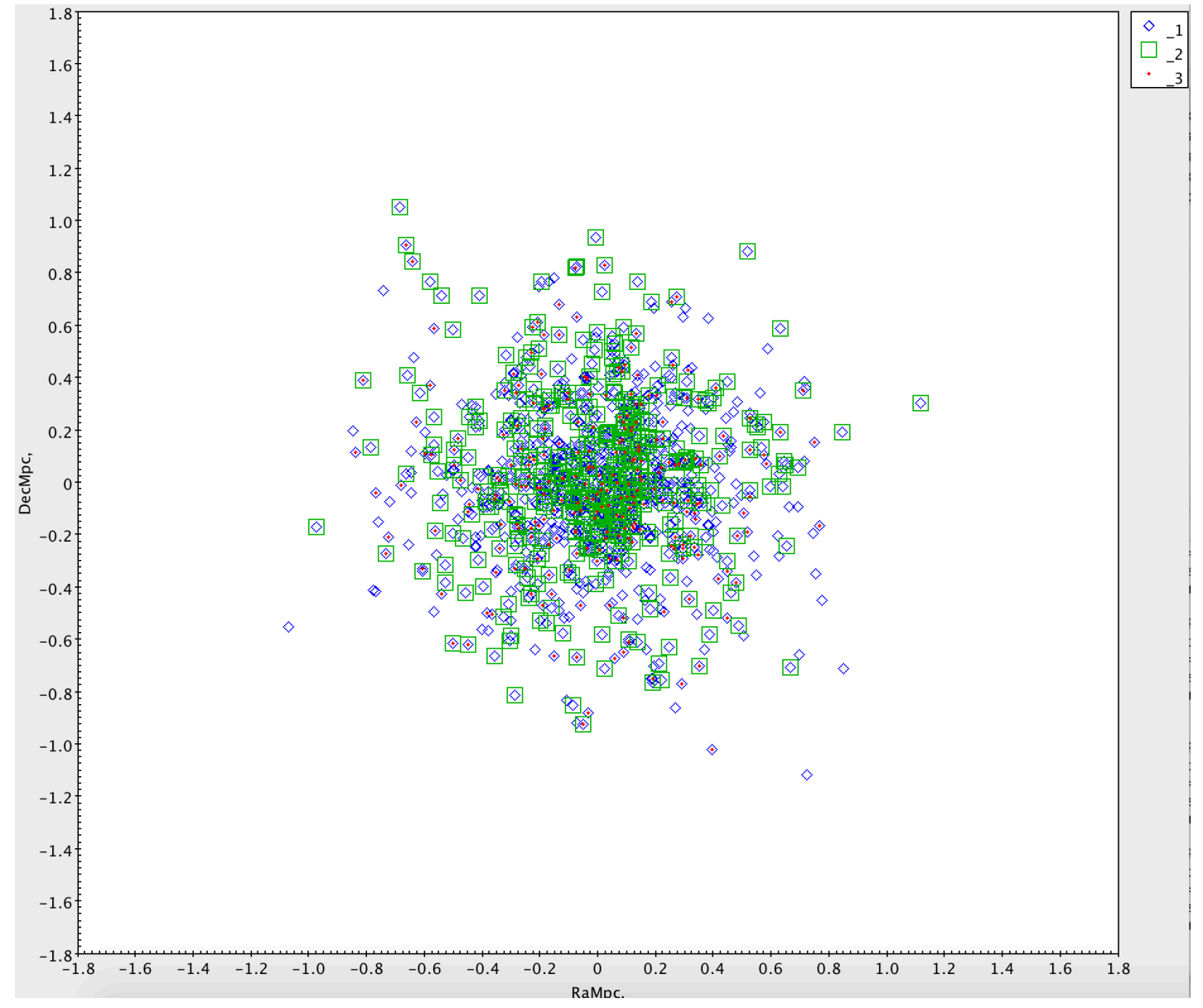



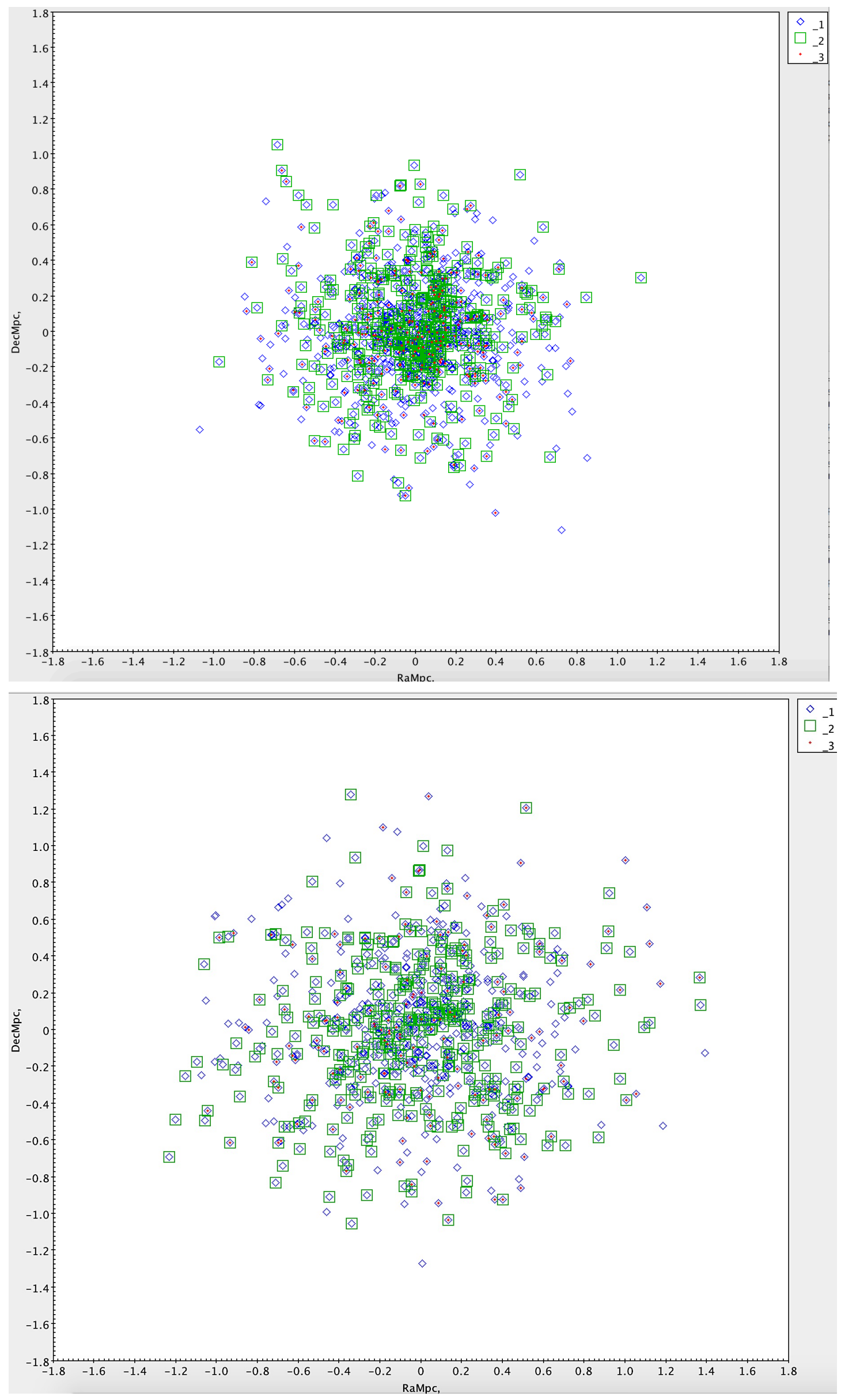


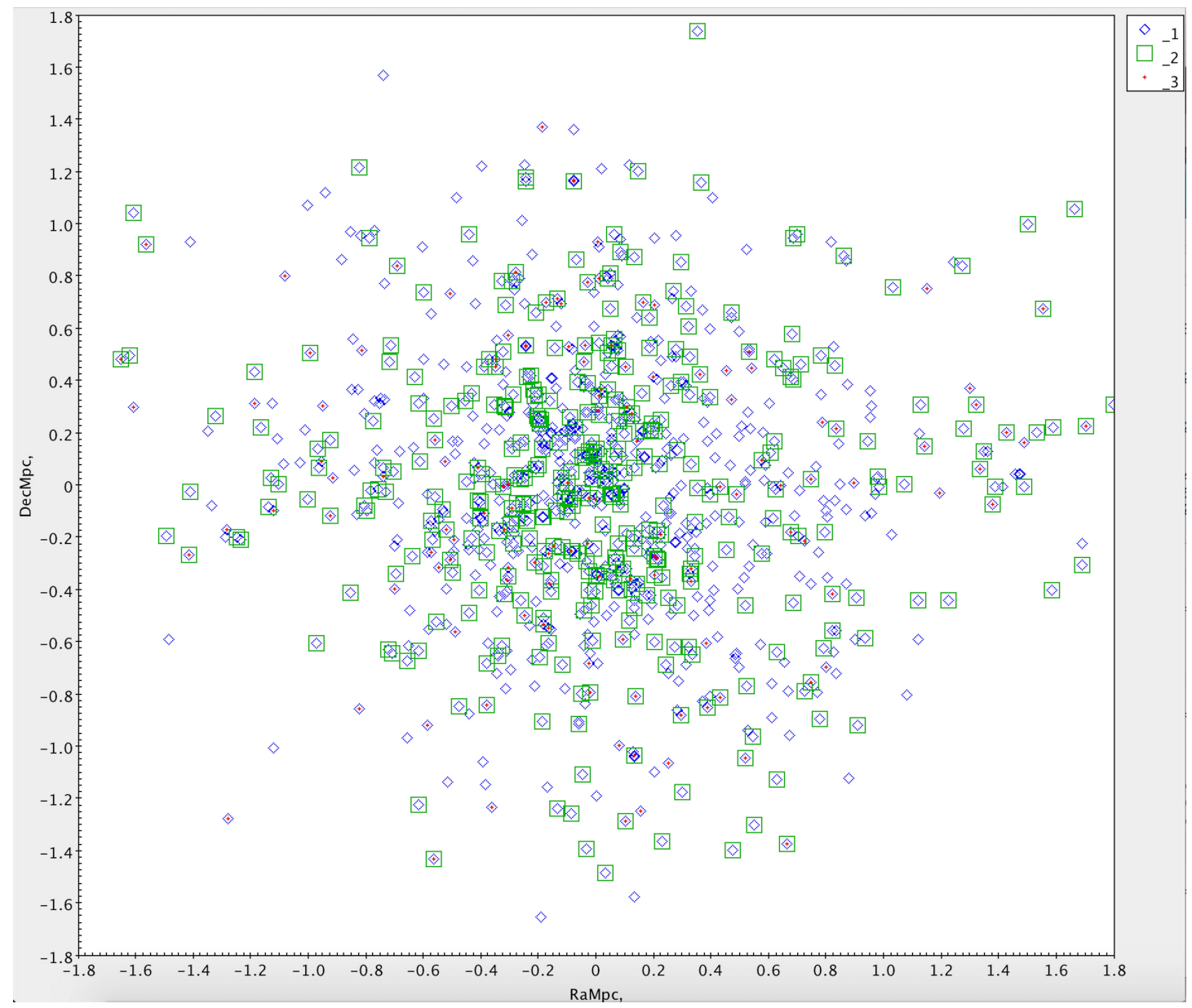

Figure 2: Y-axis: Declination in Mpc [-1.8, 1.8]. X-axis: Right Ascension in Mpc [-1.8, 1.8]. From top: all groups with 4-5 members, all groups with 6-9 members, all groups with 10-19 members, all groups with 20-50 members. The blue diamonds represent SDSS detections, the red crosses HI detections, and the green squares UV detections around the center of each galaxy group at the origin. The smallest groups remain tight around the group center, we presume the cause of this to be even smaller membership: with less there is much less gravitational pull allowing for galaxies to be at larger radii. While remaining fairly evenly concentrated throughout in the optical the UV detections throughout all bins (see Figure 3), HI detection concentration goes up in smaller membership groups and down with larger membership groups. With fewer galaxies there can be less gravitational power that would cause depletion of HI reserves via stripping and galactic interactions that occur with more galaxies.

3a. UV Analysis from GALEX: Plots that represent the NUV-r color as a function of $r$ magnitude show that many galaxies of higher $r$ magnitude do not have high detections in the NUV-r color. Galaxies which have a large population of stars will have higher UV magnitudes, which based on the plotted data means that galaxies with a higher stellar population tend to have lower magnitude in the visible spectrum, represented by $\mathrm{r}$ magnitude. Our plot (Figure1) shows a heavy concentration of galaxies with higher rband magnitudes and low NUV-r band magnitudes (the blue cloud). This leads us to conclude it plausible that group environments for galaxies cause a rapid surge in star formation before stripping member galaxies of $\mathrm{HI}$ and ending star formation.

3b. Spatial Analysis: Using the same UV data from GALEX we pursued to identify any pattern between the distance in Mpc from the center of the group and the galaxies that where 


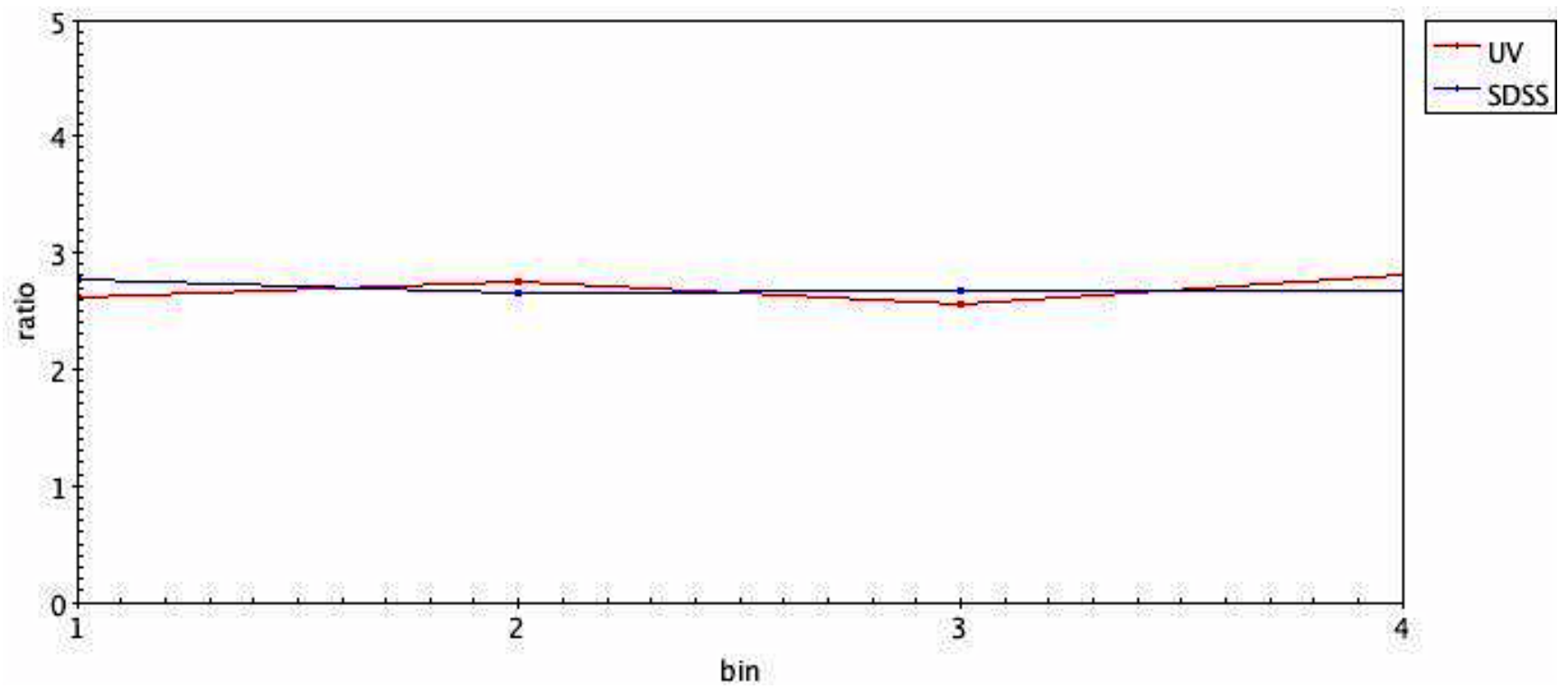

Figure 3: The four bins based on membership are indicated on the $\mathrm{x}$-axis as integers $1-4$. The concentration of UV detections is indicated as the red line while SDSS detection concentration is indicated in blue. The concentration was found by dividing two crucial radii: the radius ' $x$ ' from the group center at which $75 \%$ of galaxies detected are within said distance from center, and the radius at which $25 \%$ of galaxies detected are within said distance from center ' $x$ '. Then for each bin, I divided the $75 \% \mathrm{x}$-radii by the $25 \%$ radii for both UV and SDSS detections to find the concentrations. Both UV and SDSS concentrations remain pretty evenly distributed throughout all bins while the concentration of $\mathrm{HI}$ detections has an inverse relationship with number of galaxies in a group, as found in Hess\&Wilcots 2013.

detected in the UV. We see in Figures 2 that galaxies toward the higher density areas of the group are more likely to be UV detected, and have a higher stellar population. This shows the tidal forces, which are stronger in higher density environments, indeed have an effect on galaxies causing jarring of gas reserves leading to star formation. However, we found no correlation between group membership and the concentration of UV detections near the center. We then looked at the HI data from ALFALFA as a function of spatial distribution, similar to in Hess\&Wilcots 2013. This resulted in finding that the concentration of HI detections of galaxies near the center has an inverse relationship with the number of members in a group, and also that as a group grows in members the spatial distributions of $\mathrm{HI}$ detections increases (Hess\&Wilcots 2013). There exists less HI detected galaxies near the center due to the increase tidal and gravitational strengths from the increased membership. For a more in depth analysis of the HI detection patterns please see Hess\&Wilcots 2013.

\section{Conclusion}

A large picture of galaxy evolution can be formed from the HI data analysis described in Hess\& Wilcots 2013 combined with the UV detection analysis made in this paper. Based on the results described earlier and displayed in the plots the following are supported: galaxies in groups begin with a rapid star formation rate (blue cloud) due to tidal forces of the group as seen by the UV analysis both in the spatial distribution and the NUV-r color and $r$ magnitude. Near this time galaxies also begin being stripped of $\mathrm{HI}$ mass as seen in the spatial distributions and discussed in Hess\&Wilcots 2013 (both effects intensified the closer a galaxy is to the center of the group as gravitational and tidal forces increase with the density of galaxies in a area) as they move 
towards becoming more characteristic of red sequence older and quenched galaxies. These statements validate the theory of quenching by means of HI depletion through the forces of a galactic group along with the idea that star formation rate has inverse proportionality with distance in Mpc to the center of a group.

\section{References}

[Hess and Wilcots,2013] Evolution in the HI Gas Content of Galaxy Groups: Pre- Processing and Mass Assembly in the Current Epoch The Astronomical Journal

http://arxiv.org/abs/1308.4646

[Schawinski and Urry et al.] The green valley is a red herring: Galaxy Zoo reveals two evolutionary pathways toward quenching of star formation in early and late type galaxies. Advance Access publication http://arxiv.org/abs/1402.4814

[Haynes et al..] The Arecibo Legacy Fast ALFA Survey: The $\alpha .40$ HI Sourse Catalog, its Characteristics and their Impact on the Derivation of the HI Mass Function. The Astronomical Journal http://arxiv.org/abs/1109.0027

Haynes, Martha. "The Arecibo Legacy Fast ALFA Survey." The Arecibo Legacy Fast ALFA Survey. 1 Apr. 2013. Web. 1 June 2014. http://egg.astro.cornell.edu/index.php/ 